\title{
EchoGéo
}

30 | 2014

Varia

\section{La formation professionnelle au développement urbain : l'expérience du Groupe Huit}

\section{Benjamin Michelon}

\section{(2) OpenEdition \\ 12 Journals}

\section{Electronic version}

URL: https://journals.openedition.org/echogeo/14080

DOI: 10.4000/echogeo. 14080

ISSN: 1963-1197

\section{Publisher}

Pôle de recherche pour l'organisation et la diffusion de l'information géographique (CNRS UMR 8586)

\section{Electronic reference}

Benjamin Michelon, "La formation professionnelle au développement urbain : l'expérience du Groupe Huit", EchoGéo [Online], 30 | 2014, Online since 02 February 2015, connection on 31 July 2021. URL: http://journals.openedition.org/echogeo/14080 ; DOI: https://doi.org/10.4000/echogeo.14080

This text was automatically generated on 31 July 2021.

EchoGéo est mis à disposition selon les termes de la licence Creative Commons Attribution - Pas d'Utilisation Commerciale - Pas de Modification 4.0 International (CC BY-NC-ND) 


\title{
La formation professionnelle au développement urbain : l'expérience du Groupe Huit
}

\author{
Benjamin Michelon
}

\section{Introduction}

1 Cet article présente le partenariat entre un bureau d'études, le Groupe Huit, et une faculté de géographie, celle de l'Université de Paris 1, dans un objectif de formation professionnelle. Il témoigne par la même occasion de l'évolution des approches dans le monde de l'expertise urbaine. En effet, l'établissement de ponts entre le monde académique et le monde des praticiens est plus que jamais nécessaire. Il permet de remettre sans cesse en cause les façons de voir et de faire la ville pour améliorer et innover dans la définition et la mise en œuvre des projets urbains. Loin d'être seulement un passage de relais et un galop d'essai pour les étudiants, les ateliers proposés par le Groupe Huit constituent un facteur important d'imprégnation de l'évolution des concepts et des visions du monde véhiculés par les nouvelles générations. Lesquelles seront amenées, demain, à perpétuer et à renouveler les pratiques urbaines aujourd'hui à l'œuvre.

\section{Le Groupe Huit : un bureau d'études en Tunisie sur l'urbain}

2 Le Groupe Huit est créé en 1967 en Tunisie. Pendant 10 ans, les fondateurs de ce bureau d'études y réalisent de nombreuses études d'aménagement du territoire et d'urbanisme, en particulier dans les villes de la région phosphatière de Gafsa (Métaloui, Redeyef et Moularès). Les premiers projets concernent ainsi le Plan directeur de Gafsa, une Étude Régionale sur les Mines de Phosphate, l'Étude d'Armature urbaine de la Tunisie, un Plan directeur des Villes-Gouvernorats, etc. Ces différentes réalisations permettent au 
Groupe Huit de connaître une notoriété et une reconnaissance de son expérience. À la demande de la Banque mondiale, qui prend alors conscience du potentiel de développement des centres urbains, le champ d'action du Groupe Huit s'étend. Les fondateurs nourrissent leurs actions des réflexions issues de leurs expériences du terrain et de leurs visions ${ }^{1}$.

3 En effet, en 1975, McNamara, président de la Banque mondiale depuis 1968, prononce un discours au conseil des gouverneurs de la Banque mondiale dans lequel il fustige le fait que «les frustrations que connaissent les citadins pauvres ont de tout temps été exploitées par des extrémistes. Par conséquent, si les villes ne s'attaquent pas sans tarder et de façon plus constructive au problème de la pauvreté en milieu urbain, c'est cette dernière qui, finalement, les anéantira ». En 1976, la première conférence met l'accent sur le développement urbain et commence à faire émerger de nouvelles approches. Il s'agit alors de rompre avec l'idée selon laquelle en schématisant, la ville était définie, dessinée et produite par le pouvoir qui détient la raison et que le logement ainsi que la ville sont produits par des filières modernistes. Cette période correspond au début de la prise de conscience de l'environnement et du développement durable; la reconnaissance de la vitalité et de l'importance des productions populaires et de la participation des habitants ; la réaffirmation des droits et du droit au logement en particulier. Dans la foulée, une institution, l'ONU-Habitat, est créée en 1978 afin de rendre ces décisions opérationnelles.

4 L'installation du Groupe Huit à Paris à partir de 1977 coïncide avec ces changements. Le bureau d'études est alors sollicité pour réaliser ses premiers projets à l'international : il participe aux premiers projets de développement urbain financés par la Banque mondiale (Mali, Haïti). Il intervient au Maghreb (Maroc, Algérie), en Afrique subsaharienne (Mali, Djibouti, Burkina, Zaïre, Madagascar) et aux Caraïbes (Haïti). Cette activité se développe progressivement au cours de la décennie 80, qui voit le processus de décentralisation à l'œuvre dans les villes en développement alors que, dans le même temps, la croissance urbaine des villes africaines connaît un boom démographique sans précédent.

5 Dans les années 90, le Groupe Huit diversifie ses interventions dans un plus grand nombre de pays et notamment en Chine (Schéma directeur de Shanghai, Projet multi-cities du Zhejiang, Projet Urbain et Environnemental de l'Anhui et du Shandong, etc.). Depuis 2003, dans un contexte de concurrence internationale accrue, le Groupe Huit consolide son équipe et ses activités : il rejoint le groupe KERAN (ex groupe SCE) afin de valoriser et d'accroître son champ d'action. Il développe l'assistance technique moyen - long termes au service de projets de développement urbain dans diverses parties du monde (notamment en Afrique Sub-saharienne, au Maghreb, en Asie) tout en conservant ses compétences dans la compréhension des changements urbains qui font du Groupe Huit un précurseur dans ce domaine.

\section{Faire la ville aujourd'hui}

6 L'un des défis quotidiens auxquels est confronté le Groupe Huit reste la compréhension et la caractérisation des évolutions urbaines. En effet, comme tout bureau d'études, il doit travailler dans des contextes de plus en plus difficiles à cerner et à caractériser. Pour des raisons structurelles (faiblesse chronique des institutions statistiques), les pays d'Afrique sub-saharienne ne disposent pas de données fiables sur l'organisation de 
leur territoire et les relations qui existent entre les différents pôles de peuplement (notamment villes et bourgs). Ces données sont tout à fait courantes dans les pays du nord (recensement, analyse des migrations, mobilité résidentielle) mais inexistantes dans les pays en développement. Or la mise en place de projets de développement dans ces pays nécessite la collecte et l'analyse de ces données afin de comprendre la structuration des territoires et leurs évolutions. Pour répondre à ces enjeux, le Groupe Huit est donc amené à développer des outils adaptés. Il initie alors de nouvelles approches $^{2}$ (contrats de ville, outils de gestion urbaine et municipale tels que l'adressage ${ }^{3}$ ). Depuis sa création, il a développé des outils d'inventaire, de planification et d'aide à la décision lui permettant de s'adapter à la complexité et aux mutations des villes et des territoires tels que l'Inventaire pour la Programmation des Infrastructures et des Équipements (IPIE), le Plan Urbain de Référence (PUR), une méthode et un logiciel d'"Adressage" (URBADRESSE) et un logiciel et une méthode de planification et de gestion des réseaux routiers urbains (CITYVIA).

7 Cependant, ces outils nécessitent d'être sans cesse renouvelés. Les mutations traversant les sociétés urbaines obligent à repenser l'approche de l'évaluation urbaine et, par là même, de ses outils méthodologiques:

«Le monde devient plus incertain, imprévisible, complexe, interdépendant, parce que l'action routinisée déclinant des procédures, type planification réglementaire permettra de moins en moins d'agir de façon adaptée. En urbanisme, les situations sur lesquelles nous travaillerons seront de plus en plus singulières dans le temps comme dans l'espace. D'où la nécessité de refonder des concepts obsolètes que nous utilisons, et dessiner des pistes possibles pour l'urbanisme d'après-crise pour l'instant encore balbutiant ${ }^{4}$.

8 Alors que, selon les Nations Unies, $50 \%$ de la population mondiale est urbaine depuis 2007, les villes sont aujourd'hui reconnues comme des points névralgiques du développement et doivent donc se montrer socialement attractives et économiquement compétitives. À cet égard, le rapport annuel de la Banque mondiale en 2009 démontre que les grandes concentrations urbaines sont la condition première du développement dans les pays du Sud: «aucun pays ne s'est hissé dans la catégorie des pays à revenu intermédiaire sans s'industrialiser et s'urbaniser », et "la ruée vers les villes que connaissent les pays en développement semble chaotique, mais elle est nécessaire ${ }^{5}$. L'agglomération se trouve ainsi propulsée au cœur des gains de productivité et des économies d'échelle. Inscrite dans le cadre de la théorie de la ville globale, cette évolution tend aujourd'hui à projeter, au Nord comme au Sud, une forme urbaine spécifique, la ville diffuse, allant de pair avec la globalisation. Cette dernière conduit à une urbanisation massive et large, un étalement urbain qui a un coût important pour la collectivité. Elle remet en cause l'organisation traditionnelle de la cité sans pour autant proposer un nouveau mode de gestion collectif, se basant sur des affinités et des choix individuels. De ce fait, l'urbanisation des villes en développement reste aujourd'hui un «work in progress ${ }^{6}$ et la planification urbaine apparait donc comme une urgente nécessité.

9 Si les villes constituent des foyers de développement, de nombreuses mutations sont à l'œuvre et doivent être prises en compte pour mieux lire et planifier la ville de demain. De nouvelles stratégies d'organisation, de modernisation et de développement du territoire qui ne peuvent plus simplement se fonder sur des tableaux de bord prenant en compte des données quantitatives doivent se mettre en place. Au contraire, ces 
stratégies devront reposer sur une vision opérationnelle de l'urbanisation, fortement dépendante des acteurs présents sur le territoire et répondre à des impératifs nouveaux et à une lecture nouvelle intégrant, notamment, les informations fournies par les big datas.

C'est pourquoi le Groupe Huit recherche les contacts avec le monde de la recherche et de l'université pour enrichir ses pratiques à travers le regard critique et neuf porté par des étudiants et des chercheurs. Dans cette optique, le Groupe Huit s'est impliqué dans l'organisation d'ateliers avec des étudiants. Il s'agit d'un retour d'expérience qui favorise la confrontation entre le savoir géographique des stagiaires et le savoir professionnel que nous donnons et qu'ils doivent intégrer. Cette expérience de partage et d'échange est menée notamment dans le cadre d'un Master 2 Pratiques du développement à l'Université Paris I/Paris VII dans lequel l'équipe du Groupe Huit intervient depuis 2013 dans un cycle de cours sur le développement urbain.

\section{Le premier atelier : « si Kinshasa nous était conté »...}

11 Le premier atelier visait à proposer des scénarios de développement de la ville de Kinshasa à l'horizon 2035. Ce travail s'inscrivait dans le prolongement de l'élaboration du Schéma d'Orientation Stratégique de l'Agglomération Kinoise (SOSAK) à l'horizon 2025 qui a été confié au Groupe Huit. Ce document vise à définir les axes de développement prioritaires et à encadrer les actions en cours pour rationaliser la croissance urbaine de Kinshasa. L'objectif principal est de « renforcer l'attractivité et la compétitivité de la ville » tout en « améliorant les conditions de vie des plus pauvres ».

Il s'agissait au cours de cet atelier de demander aux étudiants de proposer des " possibles ", d'ouvrir des fenêtres en émettant des idées nouvelles et de faire émerger d'autres façons d'envisager le développement urbain. Proposer ces visions différentes permet aussi de se libérer de la contingence temporelle qui empêche parfois les élus d'avoir une vision à long terme qui dépasse la durée de leur mandat.

Donner corps à des visions d'aménagement de la ville à travers des scénarios peut ainsi favoriser une prise en compte par les élus kinois d'un futur possible. Il ne s'agit donc pas de donner un " prêt à penser » aux cadres dirigeants des pays du Sud, mais de leur donner les moyens à la fois de se positionner par rapport à ces ensembles d'idéaux types et de se représenter par là même les éléments et les évènements auxquels ils devront faire face. L'objectif étant pour eux de maîtriser le devenir de leurs villes et d'améliorer le quotidien des citadins qui y vivent, en fonction de leur vision politique et du modèle de société auquel ils aspirent.

Ce mode d'action a pour but également d'essayer de débloquer certains archétypes et modèles importés, reproduits de villes en villes, selon une vision occidentale «moderniste $»^{7}$ non questionnée et non remise en cause. La difficulté réside ainsi dans la production non pas de modèles de villes stricts et intangibles mais de véritables lignes directrices, qui permettent de s'adapter aux réalités locales tout en poursuivant des idéaux nécessaires.

15 Pour ce faire, il a été demandé aux étudiants de ne pas prendre en compte les contingences matérielles et financières que le commanditaire nous imposait. Leur démarche n'avait pas à intégrer les contraintes du bureau d'études. Cette idée pourrait paraître doublement paradoxale. En effet, en général, les géographes doivent inscrire 
leur démarche dans un terrain concret et dans des pratiques locales. De plus, cette approche pouvait paraître aller à l'encontre du processus de formation et d'apprentissage d'étudiants souhaitant découvrir, à travers cette exercice, les réalités et les contraintes du monde professionnel. Pour autant, le choix de cette démarche était nécessaire pour proposer un exercice "en chambre " à réaliser dans un délai imparti restreint. En outre, elle visait à construire et déconstruire une vision de la ville, en demandant aux étudiants de faire ressortir leurs connaissances de la ville et des problématiques urbaines. Même si les éléments de contexte n'étaient pas totalement pris en compte, il s'est agi de souligner, au final, l'importance de ces facteurs locaux pour la mise en œuvre d'un programme, notamment avec la question de la gouvernance et des choix politiques qu'il convient d'éclairer dans ce cadre de planification urbaine.

Dans un premier temps, les étudiants ont dû repérer les dynamiques urbaines qui ont contribué au développement de la ville. Il fallait prolonger la réflexion à travers la définition d'un scénario au fil de l'eau (sans intervention) afin que les étudiants repèrent les difficultés, les faiblesses, mais également les atouts de la ville de 8,7 millions d'habitants.

Dans un second temps, il était demandé aux étudiants de penser Kinshasa en fonction de différents profils urbains proposés par le Groupe Huit: ville autonome, ville inclusive, ville compétitive, ville compacte et résiliente, ville verte. Sur la base d'une rapide comparaison avec l'évolution des villes comparables', les étudiants devaient alors donner corps à des théories sur la ville et proposer des visions utopistes de Kinshasa.

Le résultat proposé par les étudiants a été à la hauteur de nos espérances : ils ont ainsi proposé quatre scénarios incluant de nombreuses idées de projets, de thématiques d'intervention en spatialisant leurs propositions pour donner corps à ces visions urbaines. Du côté du commanditaire, ces éléments ont été présentés aux techniciens de Kinshasa pour engager des discussions. Cependant, les contingences matérielles et la reproduction de modèles occidentaux ont vite repris le dessus et n'ont pas permis d'exploiter de manière optimale les résultats de ce travail.

Du côté du Groupe Huit, ce travail a constitué un temps fort dans le questionnement de nos approches, à la fois sur les objectifs d'un tel document (en revenant sur les bases d'un travail méthodologique de planification, en questionnant de nouveau les objectifs de notre travail, ses limites mais aussi ses apports) et sur la vision du développement urbain et des projets qui peuvent être mis en œuvre. Ces éléments ont été importants.

\section{Le second atelier : « l'éco-quartier », un « modèle importé ? "...}

20 Face au modèle éco-quartier qui se développe et fait l'objet maintenant de demandes tant de bailleurs que des autorités du Sud, le Groupe Huit souhaitait pouvoir se forger une opinion sur ce nouveau paradigme afin de comprendre les raisons d'un tel engouement dans les pays du Sud pour un objet occidental à la base. Il s'agissait ainsi de voir comment ce dernier avatar de la «modernité » était perçu dans les pays du Sud et comment on pouvait répondre concrètement à une telle demande. 
21 Il a donc été demandé au groupe d'étudiants de définir l'éco-quartier selon les standards européens (et sur la base d'observations réalisées dans différents écoquartiers), puis d'élaborer une liste la plus exhaustive possible des sites et des projets d'éco-quartiers et de villes nouvelles en Afrique. De plus, ces projets devaient être caractérisés d'un point de vue technique selon les informations disponibles: terminologie, principes affichés (ville verte... ou autres thématiques sur lesquelles se développe l'éco-quartier), site (situation, superficie), mode de gestion (financement), population cible, qui ont permis la réalisation de tableaux proposant un certain nombre de critères de comparaison.

Ce travail a été réalisé sur la base d'une recherche internet et d'entretiens auprès d'acteurs intervenant dans la coopération ou dans le développement urbain en France. Il leur a permis de développer une vision des éco-quartiers, terme assez large pour renfermer toutes sortes de critères et thématiques (tels que le recyclage et le tri des déchets). Les étudiants ont donc souhaité donner leur propre vision du concept d'écoquartier pour y glisser les éléments de définition indispensables à leurs yeux. Pour eux le terme d'éco-quartier n'est rien d'autre qu'une façon de plus de faire la ville, tout en surfant sur une mode actuelle.

Cette idée "d'enveloppe» conceptuelle (dans laquelle s'intègrent des éléments nécessaires à la définition d'un habitat durable) leur a permis de construire et d'introduire ce qu'ils considéraient comme essentiel dans la définition d'un quartier, en prenant particulièrement en compte le contexte africain. Les étudiants ont souligné le fait que l'éco-quartier ne prend concrètement forme qu'après avoir été mis en lien avec les enjeux et les particularités d'un territoire. Ils concluent sur le fait que «si les Africains se saisissent de cet « effet de mode » d'éco-quartier de manière stratégique, ils peuvent impulser, si cela est bien fait, le développement durable d'une ville, africaine post-moderne, à même d'accueillir les nouveaux habitants et de relocaliser les habitants des bidonvilles ». Ils ont ainsi pu toucher de près les avancées et les limites du concept, tout en montrant qu'il était nécessaire aujourd'hui d'opérationnaliser un discours visant au renouvellement des pratiques urbaines.

En conclusion, ces deux exercices ont permis des échanges fructueux. Pour les étudiants, cela a été l'occasion de comprendre la manière dont se fabrique la ville à travers la compréhension de l'évolution historique des villes et des concepts, la prise en compte des difficultés, qu'elles soient politiques, sociales ou économiques, auxquelles sont confrontés les producteurs du tissu urbain. Ils ont ainsi pu mieux appréhender la complexité du développement urbain.

En parallèle, ils ont également apporté un regard neuf sur la vision de ces processus. Devant la boulimie terminologique, ils ont su proposer des idées et des innovations qui s'inscrivent parfaitement dans la volonté du Groupe Huit de poursuivre la réflexion sur le futur des villes en développement. L'aventure n'est donc pas terminée et le Groupe Huit souhaite continuer à essaimer cette pratique de l'échange d'expériences et ce croisement de regards entre des étudiants avides d'expérience concrète et des professionnels cherchant sans cesse à prendre du recul, avec un regard critique sur leurs actions pour améliorer leurs pratiques et affiner leurs connaissances. 


\section{NOTES}

1. Poncet J., 1974. La géographie et les leçons du «Groupe Huit » en Tunisie. Espace géographique, tome $3 n^{\circ} 4$, p. 296-298.

2. Godin L., 1987. Préparation de Projets de Développement Urbain. Banque mondiale.

3. Farvacque-Vitkovic C., Godin L., Leroux H., Verdet F., Chavez R., 2004. Adressage et Gestion des Villes. Banque mondiale, collection Le développement en marche, $272 \mathrm{p}$.

4. Guigou B., 2011. Quels futurs usages de la recherche urbaine ? Les Cahiers de l'IAU îdF, Vol. 158, Juin 2011. Entretien avec Jean-Marc Offner et Alain Bourdin.

5. World Bank, 2009. World Development Report 2009: Reshaping Economic Geography. Washington , World Bank.

6. Simone A.M., 2002. Principles and realities of urban governance in Africa. Nairobi, UN Habitat.

7. Intégrant notamment un haut degré de technicité et d'expertise.

8. Cette comparaison visait à prendre en compte, notamment, le cas de Douala et de Kigali, villes étudiées dans le cadre de la thèse de Benjamin Michelon.

\section{AUTHOR}

\section{BENJAMIN MICHELON}

Benjamin Michelon, benjamin.michelon@groupehuit.com, Socio-urbaniste, travaille depuis 2012 pour le Groupe Huit. Titulaire d'une thèse sur la planification urbaine à Douala et à Kigali, récompensé en 2012 par le Prix de la thèse sur la ville, il continue à être chercheur associé à la Communauté d'études pour l'aménagement du territoire (CEAT) de l'École Polytechnique Fédérale de Lausanne (EPFL). Il est également vice-président de l'association AdP villes en Développement rassemblant des professionnels de la planification et de la gestion urbaines travaillant dans les pays émergents et en développement. Ce parcours et ces engagements lui permettent aujourd'hui de promouvoir au sein de ce bureau d'études un intérêt pour le monde de la recherche et l'enseignement académique. C'est dans ce cadre que s'inscrit cet engagement et ancrage du Groupe Huit dans des pratiques d'enseignement et de partenariat avec le monde universitaire. 